\title{
Examining the Cognitive Effects of a Special Extract of Bacopa Monniera (CDRI 08: KeenMind): A Review of Ten Years of Research at Swinburne University
}

Con Stough ${ }^{1}$, Andrew Scholey ${ }^{1}$, Vanessa Cropley ${ }^{1}$, Keith Wesnes ${ }^{1}$, Andrea Zangara ${ }^{1}$, Matthew Pase ${ }^{1}$, Karen Savage $^{1}$, Karen Nolidin ${ }^{1}$, Justine Lomas ${ }^{1} \&$ Luke A Downey ${ }^{1,2}$

${ }^{1}$ Swinburne Centre for Human Psychopharmacology; Swinburne University; Hawthorn, Melbourne.

${ }^{2}$ Department of psychology, Swansea University, Wales, UK.

Received, May 21, 2013; Accepted, June 6, 2013; Published, June 9, 2013.

\begin{abstract}
Bacopa monnieri (EBm), an Indian aquatic herb, has been used in traditional Ayurvedic medicine for centuries for indications related to memory and inflammation. More recently specific extracts of EBm have emerged that have been subjected to rigorous in vitro, animal and now human clinical trials. In this paper we discuss some of these studies with special reference to mechanisms and efficacy of a special extract of Bacopa (CDRI 08). Studies using this extract indicate that CDRI 08 has several modes of action on the human brain. Promising indications for use in humans include improving cognition in the elderly and in patients with neurodegenerative disorders.
\end{abstract}

This article is open to POST-PUBLICATION REVIEW. Registered readers (see "For Readers") may comment by clicking on ABSTRACT on the issue's contents page.

\section{INTRODUCTION}

The purpose of this paper is to introduce promising neurocognitive research on the Indian herb Bacopa monnieri and specifically an extract of this herb (CDRI 08) to the attention of the wider scientific community. There is currently a concerted effort in the field of neuropsychopharmacology to develop cognitive enhancing drugs for patient and other groups. Research on CDRI 08 constitutes such an approach. Across ten years of research we describe mechanistic and efficacy studies relating to the cognitive enhancing prospects of this specific extract.

\section{What is Bacopa monnieri?}

Bacopa monnieri Extract of Bacopa monnieri (EBm) Lin (Brahmi: Scrophulariaceae) has been used in traditional Ayurvedic medicine for various indications including memory decline, inflammation, pain, pyrexia, epilepsy and as a sedative (1). EBm contains Bacoside $\mathrm{A}$ and Bacoside B which are steroidal saponins believed to be essential for the clinical efficacy of the product. CDRI 08 or KeenMind is a high quality extract of Bacopa Monnieri containing 55\% bacosides (based on spectrophotometry) has been thoroughly studied in the Central Drug Research Institute (CDRI) in India. In this paper we outline in vitro and in vivo studies as well as human trials reported on cognition with special emphasis on CDRI 08 extract which we have exclusively used in our laboratories. Interpreting results of studies using different plant extracts is difficult. Unfortunately different extracts cannot be assumed to have comparative efficacy as they are grown and extracted differently and each will have their own characteristic and indeed unique constituent characterisation/ composition. Additionally lower quality extracts may differ in composition from year to year depending upon growing conditions and quality control. Whilst these are important issues for researchers studying herbal medicines they are beyond the scope of this paper to fully discuss.

\section{Past Research}

\section{In Vitro and In Vivo Research on Bacopa Monnieri extract (EBm)}

While EBm has been reported to have many actions, its memory enhancing effects have attracted most attention and are supported by the psychopharmacology literature. Behavioural

Corresponding Author: Prof C. Stough; Swinburne Centre for Human Psychopharmacology, Swinburne University, Hawthorn, Melbourne, Victoria, Australia 3122. Email: cstough@swin.edu.au 
studies in animals have shown that EBm improves motor learning, acquisition, retention, and delay extinction of newly acquired behaviour (2). Although the exact mechanisms of action remain uncertain, evidence suggests that $\mathrm{EBm}$ may modulate the cholinergic system and/or have antioxidant and metal chelating effects $(3,4)$. EBm may also have anti-inflammatory (5), anxiolytic and antidepressant actions (6), relaxant properties in blood vessels (7) and adaptogenic activity (8). Chronic administration of EBm inhibits lipid peroxidation in the prefrontal cortex, striatum and hippocampus via a similar mechanism to vitamin $\mathrm{E}(9)$. In an animal model of $\mathrm{AD}$, there was a dose-related reversal by $\mathrm{EBm}$ of cognitive deficits produced by the neurotoxins colchicine and ibotenic acid (4). In rodents, EBm inhibited the damage induced by high concentrations of nitric oxide in astrocytes (10). Memory deficits following cholinergic blockade by scopolamine were reversed by EBm treatment. In animal studies EBm reduced lipid peroxidation induced by $\mathrm{FeSO}_{4}$ and cumene hydroperoxide indicating that, similarly to the chelating properties of EDTA, it acts at the initiation level by chelating $\mathrm{Fe}^{++}$(11). More recently in transgenic mice, EBm supplementation reduced specific amyloid peptides by up to $60 \%$ whilst also improving memory performance (12). Thus EBm appears to have multiple modes of action in the brain all of which may be useful in ameliorating cognitive decline in the elderly or in improving cognition in patients with Alzheimer's Dementia. These include: (i) direct procholinergic action; (ii) anti-oxidant (flavonoid) capacity; (iii) metal chelation; (iv) antiinflammatory effects; (v) increased blood circulation; (vi) adaptogenic activity; and (vii) removal of $\beta$-amyloid deposits. In contrast to antioxidants such as Vitamin $\mathrm{C}$ and $\mathrm{E}$, and pharmaceuticals such as Modafinil, the multiple actions of EBm may potentially act on several pathological changes in elderly or pathological brains in concert.

\section{Bacopa Monniera as a Cognitive Enhancer}

The mechanisms outlined above may be important in understanding human cognition and intelligence. Modification of these mechanisms may improve cognitive function. The multi-modal actions of $\mathrm{EBm}$ offer potential to improve cognitive functioning across the lifespan. In order to better evaluate the historical claims associated with the herb Bacopa Monniera and to examine whether the in vitro and in vivo studies of EBm translate into improvement in cognitive performance in humans we undertook a ten-year clinical research program in participants across the lifespan. Some of this research has been published and presented previously but a significant number of studies are still underway. These studies are now described below.

\section{Human Studies on Cognition}

The first randomized clinical trial (RCT) on cognition in healthy humans for Bacopa Monniera (CDRI 08) was conducted by Stough et al (13). In this study 46 participants consumed either $320 \mathrm{mg}$ of CDRI 08 or placebo for 90 days. Outcome measures included a battery of pen and pencil neuropsychological measures, mood and measures of anxiety and conputerised tasks of information processing. The results suggested that CDRI 08 supplementation improved information processing speed, verbal learning and decreased anxiety compared to placebo. These results were consistent with anecdotal and historical usage of the herb. Some of these results of this study was replicated by Roodenrys et al (14) (who used a different cognitive battery) and then again using different cognitive measures (Cognitive Drug Research battery) in a larger sample (15). Other extracts of Bacopa monnieri have also been studied and used in human clinical trials and these are described in a recent systematic review (16). In this review the authors argue that a significant improvement in cognition was observed in all RCTs which use a 3 month chronic duration of administration. As each study used a different battery of cognitive measures it is not possible to definitely conclude which cognitive measures are best improved by EBm.

\section{Comparison to Pharmaceutical Cognitive Enhancers}

The mechanisms of action on the brain of herbal medicines such as Bacopa monnieri are often different than pharmaceutical enhancers. Herbal medicines often require chronic administration and therefore significant time to exert their influence compared to acute effects of pharmaceutical cognitive enhancers such as amphetamines or modafinil. Despite their different mechanisms of action and treatment time to cognitive improvement, a recent attempt has been made to compare the effect size or magnitude of effect of different cognitive enhancers. Neale et al (17) compared the effect sizes of herbal medicines such as EBm and Ginseng with pharmaceuticals such as Modafinil and concluded that although the time course for cognitive improvement were different the 
magnitude of cognitive improvement were similar.

\section{Acute effects of Bacopa monnieri}

Although traditionally used as a chronic cognitive enhancer, recent studies have examined whether larger doses of CDRI 08 show acute cognitive improvements using a significantly altered testing regime designed to elicit acute cognitive effects. These experimental designs either induce fatigue or stress in participants as they complete many cognitive tasks at the same time or repeatedly complete the same tests over a longer or extended period of time. In the first study (18) using a double blind placebo controlled cross-over design the authors administered different doses of KeenMind (320mg, 640mg and placebo) on different days to participants and required them to complete a multi-tasking cognitive framework. Results indicated that acute administration of KeenMind improved performance across many of the cognitive tasks indicating an improvement in divided attention as well as within the specific cognitive domains being tested. The second study by Downey et al (19) utilised the same experimental approach as the previous study (18) except for the cognitive task in which case the Cognitive Demand Battery (CDB) cognitive tests were administered instead of the multitasking framework. Results again revealed an improvement in cognitive performance across the repeated administrations of the CDB indicating that KeenMind improved attention allocated over a longer timeframe as well as the individual cognitive domains being assessed. A number of issues remain from these studies including the exact dose that is most beneficial for acute cognitive enhancement as well as the time-course for improvement post-administration.

Therefore these results suggest that CDRI 08 may also improve cognitive function acutely, although there are fewer studies examining acute cognitive changes. Further research should examine the mode of action of EBm. Acute effects may include direct neurotransmitter changes, increase in blood flow or increases in energy metabolism. The mechanisms associated with improvement in inflammation and antioxidant status, reduction in beta amyloid and increases in metal chelation are likely to occur over longer timeframes and be more suitable to improving cognitive function in abnormal brain states or in the ageing brain using chronic dosing regimens. Therefore it may be proposed that there are different mechanisms for acute and chronic cognitive enhancement due to CDRI 08 administration.

\section{Studies Currently Underway}

Improving Cognition in the Elderly

One study (20) that has been specifically designed to address the issue of cognitive aging is the Australian Research Council Longevity Intervention Study (ARCLI, ANZCTR 12611000487910). The mechanisms by which Bacopa Monniera acts on cells appear to be promising for ameliorating cognitive decline. Research in cognitive aspects of ageing (typically in 60 to 90 year-olds) has identified consistent deficits in: reasoning and decision making; spatial abilities; perceptual-motor and cognitive speed; and most robustly memory. Longitudinal studies of aged populations illuminate the time-course of cognitive deterioration. Using 5 to 10 year re-test intervals significant decrements across most cognitive capacities become evident. A recent review of longitudinal ageing studies concludes that crystallized intelligence (e.g., factual knowledge) remains intact until late ageing whereas measures of speed, information processing and aspects of memory (e.g., working memory) are more sensitive to decline from age (21).

These psychological and cognitive changes mirror brain changes over the same period of time. As we get older changes in ventricular enlargement, reduction in gross brain volume, reductions in frontal and temporo-parietal brain volume, higher levels of cortical atrophy, and increased white matter hyperintensities can be observed (22). These changes in brain morphology unfortunately are paired often with increases in neuropathological events such as an increase in $\beta$-amyloid $(A \beta)$ protein deposition, the formation of neurofibrillary tangles and increased neuroinflammatory reactions (23-28). The later changes are also seen in neurodegenerative disorders such as Alzheimer's Dementia (AD) and other neourodegenerative disorders associated with increased age. These brain processes are not exclusive to neurodegenerative disorders such as $\mathrm{AD}$, and in fact occur in a large proportion of cognitively intact individuals as they get older. For example, in one study the proportion of people without dementia with $\mathrm{A} \beta$ deposits ranged from $3 \%$ in a $36-40$ age group to $75 \%$ in an $85+$ age group (23). Increasing age also appears to be associated with numerous microscopic insults related to oxidative stress (24). Free radicals formed in the brain produce significant cellular 
damage, and mediate processes which result in large scale neural cell death. Generation of free radicals also leads to the generation of proinflammatory molecules and a state of low level chronic inflammation, leading to further cellular damage, neurodegeneration or apoptosis (25).

With these cognitive and pathological changes associated with aging in mind, we designed a large scale research study to examine whether 12 months supplementation with EBm (CDRI 08: KeenMind) improves cognitive functioning in the elderly (20). ARCLI (20) is a randomized, placebo-controlled, double-blind, clinical trial in which 310 participants will be randomized to receive an extract of Bacopa monnieri (CDRI $08320 \mathrm{mg} /$ day), or placebo daily for 12 months. Participants will be tested at baseline and then at 3,6 and 12 months postrandomization on a wide battery of cognitive, neuropsychological and mood measures, cardiovascular (brachial and aortic systolic and diastolic blood pressures as well as arterial stiffness), biochemical (assays to measure inflammation, oxidative stress and safety) as well as genetic assessments (telomere length and Single nucleotide polymorphisms). The primary aim is to investigate the effects of CDRI 08 on cognitive performance. However secondary aims are to explore the time-course of cognitive enhancement as well as potential cardiovascular and biochemical mechanisms underpinning cognitive enhancement over the 12 months of administration.

\section{Dementia}

Given the animal work indicating changes due to CDRI 08 to inflammatory and beta amyloid levels reviewed above we hypothesised that 6 month administration of $320 \mathrm{mg}$ of CDRI 08 improves cognitive functioning in patients with Alzheimer's Dementia. This study is currently underway.

\section{Cognition, Hyperactivity and Inattention in the Young}

Recently we commenced a multi-centre trial in which 6-14 year old boys with high levels of hyperactivity or inattention are given placebo or EBm (CDRI 08) for 14 weeks. The symptoms of ADHD disorder: Hyperactivity and Inattention are the primary outcome variables but other measures including brain electrical activity are also being measured. The incidence of cognitive deficits including inattention is high in the western world and parents and practitioners are currently seeking alternative treatments to amphetamine-based medications for children. Given the cognitive enhancing potential shown for KeenMind in previous studies on healthy participants, it was hypothesised that chronic administration of KeenMind may be of assistance to children with attention problems. This study is currently underway.

\section{SUMMARY}

Ten years of research at Swinburne with the same extract CDRI 08 suggest that this extract of Bacopa monnieri is a safe and efficacious cognitive enhancer. Robust evidence for its chronic enhancing effect is strongest, with recent studies also suggesting an acute cognitive enhancing effect but with less evidence. Extracts of EBm, particularly high quality extracts such as CDRI 08 show promise as a cognitive enhancer across a range of ages but significantly more research with child and adolescent samples are required. Future research should better characterize the metabolites responsible for the cognitive changes due to supplementation. Additionally trials with longer administration durations (e.g., 5 years or longer) should be conducted to examine whether EBm may offer protection from neurodegenerative disorders.

\section{ACKNOWLEDGEMENTS}

The authors wish to thank: the Australian Research Council "Discovery Grant" (DP1093825) to CS and AS, SOHO Flordis International (SFI), and the Menzies Foundation (MP) for funding contributing to the research on CDRI 08.

\section{REFERENCES}

1. Russo A, Borrelli F. Bacopa monniera, a reputed nootropic plant: an overview. Phytomedicine, 2005; 12(4): 305-17.

2. Singh HK, Dharwan BN. Neuropsychopharmacological effects of the Ayurvedic nootropic Bacopa monniera Linn.(Brahmi). Indian Journal of Pharmacology, 1997; 29:S359-S365.

3. Agrawal A. [Thesis]. (1993). Varanasi: Banaras Hindu University

4. Bhattacharya SK, Kumar A, Ghosal S. Effect of Bacopa monniera on animal models of Alzheimer's disease and perturbed central cholinergic markers of cognition in rats. Research Communications in Pharmacology and Toxicology, 1999; 4(3\&4):1-12.

5. Jain SK. Ethnobotany and research on medicinal plants in India. Ciba Found Symp, 1994; 185:15364, discussion 164-68. 
6. Bhattacharya S, Ghosal S. Anxiolytic activity of a standardized extract of Bacopa monniera: an experimental study. Phytomedicine, 1998; 5(2):77-82.

7. Dar A, Channa S. Calcium antagonistic activity of Bacopa monniera on vascular and intestinal smooth muscles of rabbit and guinea pig. J Ethnopharmacol, 1999; 66:167-74.

8. Rai D, Bhatia G, Palit G, et al. Adaptogenic effect of Bacopa monniera (Brahmi). Pharmacol Biochem Behav, 2003;75(4):823-30.

9. Bhattacharya SK, et al. Antioxidant activity of Bacopa monniera in rat frontal cortex, striatum and hippocampus, Phytother Res, 2000; 14(3):174-9.

10. Russo A, Borrelli F, Campisi A, Acquaviva R, Raciti G, Vanella A. Nitric oxide-related toxicity in cultured astrocytes: effect of Bacopa monniera. Life Sci, 2003; 73(12): 1517-26.

11. Tripathi YB. Chaurasia S. Tripathi E, et al. Bacopa monniera Linn. as an antioxidant: Mechanism of action. Indian $J$ Exp Biol, 1996;34:523-6.

12. Holcomb LA, Dhanasekaran M, et al. Bacopa monniera extract reduces amyloid levels in PSAPP mice. J Alzheimers Dis, 2006; 9(3):243-51.

13. . Stough $\mathrm{C}$, et al. The chronic effects of an extract of Bacopa monniera (Brahmi) on cognitive function in healthy human subjects. Psychopharmacology, 2001; 156(4):481-4.

14. Roodenrys S, Booth D, Bulzoni S, Phipps A, Micallef C, Smoker J. Chronic effects of Brahmi ( Bacopa monnieri ) on human memory. Neuropsychopharmacology, 2002; 27: 279-81.

15. Stough et al. Examining the nootropic effects of a special extract of Bacopa monniera on human cognitive functioning: 90 day double-blind placebo-contrlled randomized trial. Phytomedicine, 2008; 22:1629-34.

16. Pase, M.P., Kean, J., Sarris, J., Neale, C., Scholey, A., \& Stough, C. The cognitive enhancing effects of bacopa monnieri: A systematic review of randomized controlled human clinical trials. JACM, 2012; 18 (7):647-52.

17. Neale, C., Camfield,D., Reay, J, Stough, C. \& Scholey, A. Cognitive effects of two nutraceuticals Ginseng and Bacopa benchmarked against modafinil: a review and comparison of effect sizes. British Journal of Clinical Pharmacology, 2013; in press.
18. Benson, S., Downey, L.A., Stough, C., Wetherell, M., Zangara, A., \& Scholey, A. An acute, doubleblind, placebo controlled crossover study of $320 \mathrm{mg}$ and $640 \mathrm{mg}$ doses of Bacopa monnieri (CDRI 08) on multitasking stress reactivity and mood. Phythotherapy Research; 2013; in press.

19. Downey, L.A., Murray, M., Rourke, J., Patak, B., Pase, M., Zangara, A., Scholey, A. \& Stough, C. An acute, double -blind, placebo controlled crossover study of $320 \mathrm{mg}$ and $640 \mathrm{mg}$ doses of a special extract of Bacopa monnieri (CDRI 08) on sustained cognitive performance. Phytotherapy Research; 2013; in press.

20. Stough, C., Pase, M., Cropley, V., Myers, S., Nolidin, K., King, R., Camfield, D., Wesnes, K., Pipingas, A., Croft, K., Chang, D. \& Scholey, A. A randomized controlled trial investigating the effect of Pycnogenol and Bacopa CDRI 08 herbal medicines on cognitive, cardiovascular, and biochemical functioning in cognitively healthy elderly people: the Australian Research Council Longevity Intervention (ARCLI) study protocol (ANZCTR12611000487910). Nutrition Journal, 2012; 11:1-9.

21. Christensen, H.; Kumar, R., The Ageing Brain. (2003). In: Sachdev, P. S. (eds), Swets \& Zeitlinger, B.V.: Lisse pp. 75-96.

22. Looi, J., Sachdev, P. S. (2003). In: Sachdev, P. S. (eds), The Ageing Brain. Swets \& Zeitlinger: Lisse pp. 49-62.

23. Braak, H., Braak, E., Frequency of stages of Alzheimer-related lesions in different age categories. Neurobiol Aging, 1997; 18(4): 351-7.

24. Packer, L. (1992). In: Packer, L. et al. (eds), Free Radicals in the Brain. Springer-Verlag: New York.

25. Sarkar, D., Fisher, P. B., Molecular mechanisms of aging-associated inflammation. Cancer Lett, 2006; 236(1): 13-23

26. Fydrych, A.; Moir, R.D.; Huang, C.; Shi, Y.; Rogers, J.T.; Huang, X., Amyloid-targeted metal chelation, anti-oxidative stress, and antiinflammation as potential Alzheimers therapies Current Bioactive Compounds; 2008; 4:140-9.

27. Chauhan, V., Chauhan, A., Oxidative stress in Alzheimer's disease. Pathophysiology, 2006; 13(3): 195-208

28. Akiyama, H. et al. Inflammation and Alzheimer's disease. Neurobiology of Aging, 2000; 21(3): 383421. 